\title{
Implementation of an Inhaled Nitric Oxide Protocol Decreases Direct Cost Associated With Its Use
}

\author{
Deanna R Todd Tzanetos MD MSCI, Jon J Housley PharmD MBA, Frederick E Barr MD MSCI, \\ Warren L May PhD, and Cheri D Landers MD
}

\begin{abstract}
BACKGROUND: The objective of this study was to determine whether the implementation of an inhaled nitric oxide protocol (INO) in a pediatric ICU (PICU) would reduce cost associated with its use without negatively affecting patient outcomes. METHODS: This is a retrospective cohort study of 76 subjects who required INO therapy in the PICU during the study period. A nitric oxide setup and weaning protocol was implemented in the PICU. The medical records of subjects who had received INO 18 months after protocol implementation, as well as the medical records of subjects who had received INO in the $\mathbf{1 8}$ months before protocol implementation, were reviewed. Length of time on INO, cost of INO per subject, mortality, stay, and ventilator hours were recorded. RESULTS: There were 38 subjects in the pre-protocol group and 38 subjects in the post-protocol group. There was a statistically significant decrease in the median per subject cost of INO between the pre- and post-protocol groups $(P<.01)$. There was no statistically significant difference in the median duration of INO use $(P=.06)$, median PICU $(P=.42)$ or hospital $(P=.58)$ stay, median duration of mechanical ventilation $(P=.79)$ or percent mortality $(P=.28)$ between the 2 groups. CONCLUSIONS: Implementation of an INO setup and weaning protocol in a PICU reduces the cost associated with its use without a statistically significant difference in mortality. In an era of increased awareness regarding healthcare spending, implementation of evidence-based protocols can provide a way to ensure the judicious utilization of medical resources. Key words: pediatrics; pulmonary hypertension; protocols/practice guidelines. [Respir Care 2015;60(5):644-650. @ 2015 Daedalus Enterprises]
\end{abstract}

\section{Introduction}

Like many drugs used in the pediatric setting, inhaled nitric oxide (INO) has been utilized both for United States Food and Drug Administration-approved indications as well as for off-label or investigational indications. The only current approved indication for INO is for the treat-

\footnotetext{
Dr Todd Tzanetos is affiliated with the Department of Pediatrics, Division of Critical Care, University of Louisville School of Medicine, Louisville, Kentucky. Dr Housley is affiliated with Hospital Administration, UK HealthCare, Lexington, Kentucky. Dr Barr is affiliated with the Department of Pediatrics, Division of Critical Care, and Dr May is affiliated with the Department of Medicine and Center of Biostatistics/ Bioinformatics, University of Mississippi, Jackson, Mississippi. Dr Landers is affiliated with the Department of Pediatrics, Division of Critical Care, University of Kentucky School of Medicine, Lexington, Kentucky.

Dr Barr discloses a relationship with Asklepion Pharmaceuticals; all other authors report no conflicts of interest.
}

ment of term and near-term neonates presenting with hypoxemic respiratory failure associated with pulmonary hypertension. Off-label uses include treatment of ARDS, postoperative pulmonary hypertension after congenital heart surgery, and right ventricular dysfunction following cardiac surgery or transplantation. ${ }^{1,2}$

Nitric oxide relaxes vascular smooth muscle by binding to the heme moiety of cytosolic guanylate cyclase, which in turn activates guanylate cyclase and increases levels of cyclic guanosine $3^{\prime}, 5^{\prime}$-monophosphate, ultimately resulting in decreased intracellular calcium levels leading to

\footnotetext{
Correspondence: Deanna Todd Tzanetos MD MSCI, Department of Pediatrics, Division of Critical Care, University of Louisville School of Medicine, 571 S Floyd Street, Suite 332, Louisville, KY 40202. E-mail: drtzan01@ louisville.edu.
}

DOI: $10.4187 /$ respcare. 03308 
arteriolar smooth muscle relaxation. When nitric oxide is inhaled, pulmonary vasodilation occurs, and an increase in the partial pressure of arterial oxygen occurs. This dilation in well-ventilated lung areas redistributes blood flow away from lung areas where ventilation and perfusion ratios are poor, improving ventilation perfusion mismatch. ${ }^{3,4}$

\section{See the Related Editorial on Page 760}

Prolonged use of INO can be associated with methemoglobinemia, need for continued mechanical ventilation, and increased bleeding risk. ${ }^{5,6}$ Inhaled nitric oxide is a very costly therapy. ${ }^{7}$ During the study period at our institution, the direct cost of nitric oxide was $\$ 100 / \mathrm{h}$ of use regardless of dose. The yearly cost of INO institution-wide was approximately $\$ 1.8$ million dollars. Given the high cost and risks of treatment, Barr and $\mathrm{Macrae}^{8}$ recommended that reasonable attempts to wean inhaled nitric oxide should be made every $12-24 \mathrm{~h}$, utilizing an objective weaning protocol that measures the physiologic response to the decrease in dose of INO. It is also recommended that inhaled nitric oxide should be discontinued after a short trial if no beneficial effect has been documented. ${ }^{8}$

It is known that rebound pulmonary hypertension can occur upon withdrawal of INO. This is due in part to the reduced production of endogenous $\mathrm{NO}$ and the reduced capacity to generate cyclic guanosine $3^{\prime}, 5^{\prime}$-monophosphate quickly when exogenous NO is discontinued. ${ }^{9}$ Negative feedback inhibition by exogenous NO has been postulated to account for this observation and has been demonstrated to occur in vitro for both inducible nitric oxide synthase and endothelial nitric oxide synthase. ${ }^{10}$ Slow weaning of NO may ameliorate rebound pulmonary hypertension. ${ }^{11}$ Therefore, a weaning protocol that incorporates a slow, objective weaning process is desirable.

Several studies have demonstrated that implementation of protocols in the ICU can be beneficial. Many groups have implemented standardized ventilator weaning protocols, which decrease ventilator hours. ${ }^{12-14}$ Others have implemented early goal-directed therapies for the management of septic shock, which have demonstrated improved outcomes. ${ }^{15,16}$ Given the growing body of literature to support protocols to facilitate care in the ICU, the purpose of this study was to determine whether the implementation of an INO setup and weaning protocol would reduce the cost of INO, hours on INO, ventilator hours, and hospital and ICU length of stay without an adverse effect on mortality.

\section{Methods}

This study was performed at the University of Kentucky (Lexington, Kentucky). Institutional review board approval was obtained. A nitric oxide setup and weaning protocol

\section{QUICK LOOK}

\section{Current knowledge}

Inhaled nitric oxide is a selective pulmonary vasodilator approved for the treatment of term and near-term neonates with hypoxemic respiratory failure associated with pulmonary hypertension. Off-label use in adults and pediatrics is common and expensive. Restricting use to patients with a clinically important response and limiting the duration of therapy is important to minimize costs and maximize effectiveness.

\section{What this paper contributes to our knowledge}

Implementation of an evidence-based inhaled nitric oxide implementation and weaning protocol in a pediatric ICU reduced costs without a statistically significant difference in mortality. There was a statistically significant decrease in the median per subject costs, but no difference in the median duration of use, median ICU or hospital stay, or duration of ventilator days. Early termination of therapy in nonresponders was the key to cost reduction.

were chosen for use in a 12-bed combined medical and cardiac pediatric ICU (PICU). The protocols were developed at another institution. The setup protocol was implemented to test initial response to INO (Fig. 1). If the subject was found to be a nonresponder, INO therapy was discontinued. If the subject was found to be a responder, INO was continued and the weaning protocol was utilized (Fig. 2). ${ }^{8}$ Before protocol implementation, weaning of INO was dictated by individual physicians based on their clinical judgment. A didactic lecture on the physiology of nitric oxide, indications for use, and instructions on how to perform the actions in the protocol was videotaped. The videotaped lecture was placed on the hospital's web-based training site, and all respiratory therapists were required to view the lecture and sign that they had done so. The same lecture was repeated live to all nurses working in the PICU. An order set for the nitric oxide protocol was placed in the computerized order entry system. INO per protocol was the default order unless the physician chose to opt out. Once the order was placed for INO, a paper copy of the INO setup and weaning protocols was placed at the subject's bedside and completed by the respiratory therapist carrying out the wean. After all respiratory therapists and nurses had been trained on the use of the protocol, a go-live date was chosen, and the protocol was implemented.

Using hospital billing records, subjects who had received INO in the PICU for the 18 months before and 18 months after protocol implementation were identified. PICU encounters were identified in the hospital billing records. The charge description master codes 5814700, 58209000, 


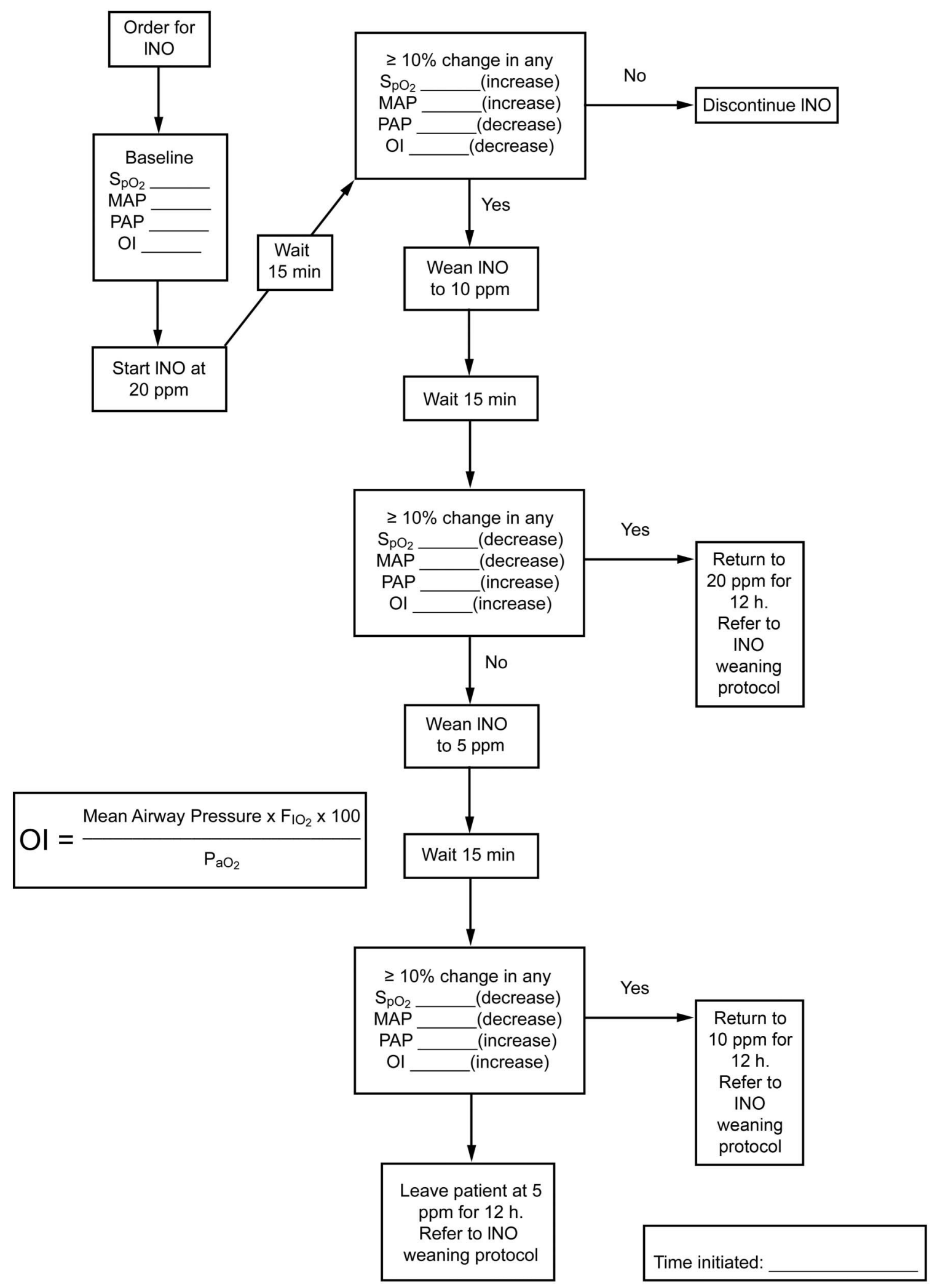

Fig. 1. Pediatric ICU (PICU) inhaled nitric oxide $(\mathrm{INO})$ setup protocol. MAP = mean arterial pressure, $\mathrm{PAP}=$ peak airway pressure, $\mathrm{OI}=\mathrm{ox}-$ ygenation index. 


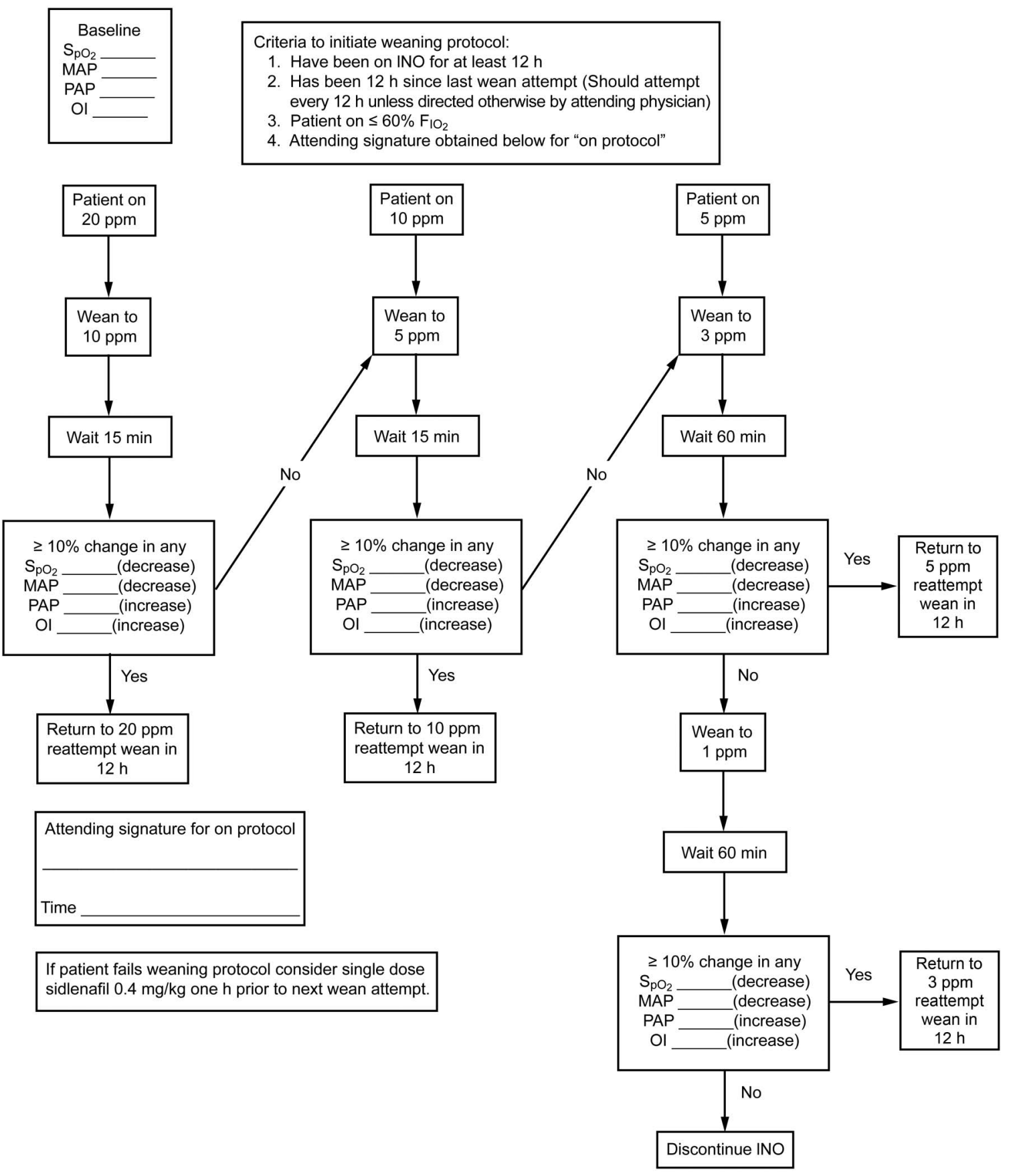

Fig. 2. Pediatric ICU (PICU) inhaled nitric oxide (INO) weaning protocol. MAP = mean arterial pressure, PAP = peak airway pressure, $\mathrm{OI}=$ oxygenation index.

and 58210000 for extensive gas delivery were used to identify subjects who had received an inhaled gas. Datacollected represent direct cost incurred by the institution for provision of INO therapy per patient. A chart review was performed to collect subject's indication for INO use (hypoxia or congenital heart disease), mortality, hours on INO, ventilator hours, hospital and ICU length of stay, and direct cost of INO.

\section{Statistical Analysis}

Categorical variables (indication for treatment, gender, and mortality) are reported as proportions and group comparisons were made using Fisher exact tests. Continuous variables (age, NO duration, cost, ICU and hospital length of stay, and ventilator hours) were noticeably skewed; therefore, we report the medians (range, minimum to max- 
Table 1. Subject Characteristics

\begin{tabular}{lccc}
\hline \hline & \multicolumn{2}{c}{ INO } & \\
\cline { 2 - 3 } & Pre-Protocol & Post-Protocol & \\
\hline Subjects, $n$ & 38 & 38 & \\
Indication (\% hypoxia) & 63 & 47 & .17 \\
Gender (\% male) & 71 & 74 & .80 \\
Age (d) & & & \\
$\quad$ Median & 4.5 & 4.4 & .95 \\
$\quad$ Range & $0-211$ & $0-5,797$ & \\
\hline
\end{tabular}

imum) for the 2 groups and used nonparametric MannWhitney U tests to compare the distributions. We consider $P<.05$ statistically significant. SAS 9.3 (SAS Institute, Cary, North Carolina) was used for all data analysis.

\section{Results}

Forty-one subjects were identified by billing records as having been in the PICU and having received an inhaled gas in the 18 months before protocol implementation. Three of those subjects were found to have received heliumoxygen mixture instead of INO and were thus not included in the analysis. Thirty-eight subjects were identified by hospital billing records as having been in the PICU and having the aforementioned charge description master codes for extensive gas delivery after protocol implementation. All 38 subjects received INO. The subject characteristics can be seen in Table 1. The median cost per subject of INO in the pre-protocol group was $\$ 9,438(\$ 1,074-66,763)$ and $\$ 4,152(\$ 1,152-63,286)$ in the post-protocol group, which was a statistically significant difference $(P<.01)$. The median duration of INO use in the pre-protocol group was $123.3 \mathrm{~h}(2.1-759.5 \mathrm{~h})$ and $45.4 \mathrm{~h}(0.3-878.9 \mathrm{~h})$ in the post-protocol group, which was not a statistically significant difference $(P=.06)$. There were 7 deaths in the pre-protocol group and 11 deaths in the post-protocol group, which was not a statistically significant difference $(P=.28)$. There was no statistically significant difference in the hours of mechanical ventilation $(P=.78)$ or stay in the hospital $(P=.58)$ or ICU $(P=.42)$. Table 2 provides a detailed summary of the results.

\section{Discussion}

The use of INO in pediatric critical care is controversial. A recent meta-analysis observed no difference associated with the use of INO in postoperative congenital heart disease patients in regards to mortality, mean airway pressure, $\mathrm{F}_{\mathrm{IO}_{2}}$, or $\mathrm{P}_{\mathrm{aO}} \cdot{ }^{17}$ Other studies demonstrate its effectiveness in the management of postoperative pulmonary hypertension in congenital heart disease patients. ${ }^{18,19}$ Large multi-center trials have demonstrated that INO reduces the
Table 2. Results

\begin{tabular}{lccc}
\hline \hline & \multicolumn{2}{c}{ INO } & \\
\cline { 2 - 3 } & Pre-Protocol & Post-Protocol & \\
\hline Subjects, $n$ & 38 & 38 & .28 \\
Death (\%) & 18 & 29 & .42 \\
ICU LOS (d) & & & \\
$\quad$ Median & 16 & 18.5 & \\
$\quad$ Range & $2-107$ & $0-246$ & .58 \\
Hospital LOS (d) & & & \\
$\quad$ Median & 27 & 25.5 & .06 \\
$\quad$ Range & $2-378$ & $0-246$ & \\
INO duration (h) & & & \\
$\quad$ Median & 123.3 & 45.4 & \\
$\quad$ Range & $2.1-759.5$ & $0.3-878.9$ & \\
Cost (\$) & & & \\
$\quad$ Median & 9,438 & 4,152 & \\
$\quad$ Range & $1,074-66,763$ & $1,152-63,286$ & \\
Ventilator time (h) & & & \\
$\quad$ Median & 312.3 & 288.7 & \\
Range & $0-2,570$ & $0.4-5,904$ & \\
\hline LOS = length of stay & & & \\
\hline
\end{tabular}

need for extracorporeal membranous oxygenation in newborns with persistent pulmonary hypertension. ${ }^{20,21}$ Given the high cost of INO and the lack of demonstrable effect on mortality, utilization of a protocol to minimize costs without adversely affecting outcomes is prudent.

In this study, we determined that the systematic implementation of an INO protocol in a PICU decreases cost associated with its use. One benefit to this particular protocol was the use of the setup protocol (Fig. 1). The setup protocol utilizes objective criteria to determine whether the subject has responded to the initiation of INO. If the subject is a nonresponder, the INO is stopped after $15 \mathrm{~min}$. If the patient is a responder, the INO is continued and the weaning protocol is implemented. The weaning protocol requires that attempts to wean INO are performed every $12 \mathrm{~h} .{ }^{8}$ The INO is weaned as much as tolerated with each attempt, ensuring that if the subject meets objective criteria for discontinuation, INO will be discontinued. The use of a setup protocol to determine whether the subject objectively improves with the addition of INO in combination with repeated attempts at weaning help to ensure that INO is not continued unless the clinical condition requires it.

There was no statistically significant difference in mortality between the pre- and post-protocol groups in this study. However, the raw data reveal $18 \%$ mortality ( 7 deaths) in the pre-protocol group and $29 \%$ mortality (11 deaths) in the post-protocol group. As presented in Table 1 , the indication for INO use, although not statistically significant, appears to have shifted from primarily hypoxia 
in the pre-protocol group to congenital heart disease in the post-protocol group. It should be noted that the protocol implementation process coincided with a revitalization of the congenital cardiac surgical program at our institution, and the complexity of congenital cardiac lesions and surgical procedures performed during this time was everincreasing. The authors feel that the increased number of deaths in the post-protocol group, although not statistically significant, likely reflects an increase in the severity of illness rather than a reflection on the use of the protocol. In fact, anecdotally, some intensivists felt the protocol was too conservative.

The median duration of INO use and ventilator hours were lower in the post-protocol group, although this was not statistically significant. Further prospective study evaluating the implications of an INO protocol on ventilator hours is warranted, as this could present an additional cost savings.

The limitations to this study include its retrospective nature and the relatively small sample size. Initially, this study began as a quality improvement project. Data were collected in a retrospective and de-identified fashion in compliance with institutional review board requirements for quality improvement projects. Only the data presented here were collected. Common severity of illness scores, such as pediatric risk of mortality scores, were not available at our institution. Indications for INO initiation were divided into 2 broad categories of hypoxia and congenital heart disease. For these reasons, it is possible that the preand post-protocol groups were composed of different patient populations with different indications for administration of INO in the hypoxia group. Given the retrospective nature of the study, it is not possible for us to be certain of protocol adherence. Thus, the decrease in cost and hours on INO could have simply been due to the Hawthorne effect, rather than to strict protocol adherence. However, these reductions are still an important result of protocol implementation. This study demonstrates that use of a protocol to drive nitric oxide administration limits hours on nitric oxide and therefore decreases costs associated with INO use with no statistically significant difference in mortality. A randomized prospective study is warranted to evaluate the use of these protocols to drive INO therapy with careful attention to safety, efficacy, adherence, and cost effectiveness.

Several studies have demonstrated that protocol implementation can improve care and increase adherence to evidence-based medicine guidelines. We utilized the approach advocated by Kollef et $\mathrm{al}^{22}$ for protocol development. Perhaps the greatest barrier to protocolizing care is the actual implementation process. In our study, we utilized a videotaped didactic lecture along with web-based training to disseminate information regarding the indications for INO use and the actual performance of the pro- tocol. We found this approach to be an efficient and effective way of targeting the information to all necessary personnel who may come in contact with a subject on the protocol. In the ICU, there are people who may work only nights or who may float into the ICU only occasionally. Utilizing the web-based training allowed us to reach all individuals who could be involved in the care of these subjects in a timely and efficient manner.

\section{Conclusions}

Implementation of a protocol to direct the administration of INO in a PICU decreased direct cost associated with INO with no statistically significant difference in mortality. In a time of increasing awareness of the cost of medicine and increased incentives to provide better care at lower costs, protocol development should likely play an important role. With systematic implementation and careful monitoring for patient safety, evidence-based protocols can streamline processes. As doctors, nurses, and other healthcare professionals become increasingly burdened with documentation requirements and large patient volumes, protocols can provide a means of providing care in a timely, evidence-based manner. Not all treatments or conditions will be amenable to protocol utilization, but with evidence-based development, systematic implementation, quality assurance controls, and frequent reviews, protocols can be a helpful adjunct in the provision of care.

\section{REFERENCES}

1. Dobyns EL, Cornfield DN, Anas NG, Fortenberry JD, Tasker RC, Lynch A, et al. Multicenter randomized controlled trial of the effects of inhaled nitric oxide therapy on gas exchange in children with acute hypoxemic respiratory failure. J Pediatr 1999;134(4):406-412.

2. Taylor MB, Laussen PC. Fundamentals of management of acute postoperative pulmonary hypertension. Pediatr Crit Care Med 2010; 11(2Suppl):S27-S29.

3. Wratney AT, Hamel DS, Cheifetz IM. Inhaled gases. In: Nichols DG, editor. Roger's textbook of pediatric intensive care, 4th edition. Philadelphia, PA: Lippincott Williams \& Wilkins;2008:532-543.

4. Cornfield DN. Acute respiratory distress syndrome in children: physiology and management. Curr Opin Pediatr 2013;25(3):338-343.

5. Nakajima W, Ishida A, Arai H, Takada G. Methaemoglobinaemia after inhalation of nitric oxide in infant with pulmonary hypertension. Lancet 1997;350(9083):1002-1003.

6. George TN, Johnson KJ, Bates JN, Segar JL. The effect of inhaled nitric oxide therapy on bleeding time and platelet aggregation in neonates. J Pediatr 1998;132(4):731-734.

7. Pierce CM, Peters MJ, Cohen G, Goldman AP, Petros AJ. Cost of nitric oxide is exorbitant (letter). BMJ 2002;325(7359):336.

8. Barr FE, Macrae D. Inhaled nitric oxide and related therapies. Pediatr Crit Care Med 2010;11(2 Suppl):S30-S36.

9. Goldman AP, Haworth SG, Macrae DJ. Does inhaled nitric oxide suppress endogenous nitric oxide production? J Thorac Cardiovasc Surg 1996;112(2):541-542.

10. Buga GM, Griscavage JM, Rogers NE, Ignarro LJ. Negative feedback regulation of endothelial cell function by nitric oxide. Circ Res 1993;73(5):808-812. 


\section{IMPLEMENTATION OF AN InHALED NitRic Oxide Protocol}

11. Assreuy J, Cunha FQ, Liew FY, Moncada S. Feedback inhibition of nitric oxide synthase activity by nitric oxide. Br J Pharmacol 1993; 108(3):833-837.

12. Marelich GP, Murin S, Battistella F, Inciardi J, Vierra T, Roby M. Protocol weaning of mechanical ventilation in medical and surgical patients by respiratory care practitioners and nurses: effect on weaning time and incidence of ventilator-associated pneumonia. Chest 2000;118(2):459-467.

13. Duan J, Tang X, Huang S, Jia J, Guo S. Protocol-directed versus physician-directed weaning from noninvasive ventilation: the impact in chronic obstructive pulmonary disease patients. J Trauma Acute Care Surg 2012;72(5):1271-1275.

14. Horst HM, Mouro D, Hall-Jenssens RA, Pamukov N. Decrease in ventilation time with a standardized weaning process. Arch Surg 1998;133(5):483-488.

15. Micek ST, Roubinian N, Heuring T, Bode M, Williams J, Harrison $\mathrm{C}$, et al. Before-after study of a standardized hospital order set for the management of septic shock. Crit Care Med 2006;34(11):2707-2713.

16. Kortgen A, Niederprüm P, Bauer M. Implementation of an evidencebased "standard operating procedure" and outcome in septic shock. Crit Care Med 2006;34(4):943-949.

17. Bizzaro M, Gross I, Barbosa F. Inhaled nitric oxide for the postoperative management of pulmonary hypertension in infants and chil- dren with congenital heart disease. Cochrane Database Syst Rev 2014;(7):CD005055.

18. Russell IA, Zwass MS, Fineman JR, Balea M, Rouine-Rapp K, Brook M, et al. The effects of inhaled nitric oxide on postoperative pulmonary hypertension in infants and children undergoing surgical repair of congenital heart disease. Anesth Analg 1998; $87(1): 46-51$.

19. Fullerton DA, Jones SD, Jaggers J, Piedalue F, Grover FL, McIntyre RC Jr. Effective control of pulmonary vascular resistance with inhaled nitric oxide after cardiac operation. J Thorac Cardiovasc Surg 1996;111(4):753-762.

20. Clark RH, Kueser TJ, Walker MW, Southgate WM, Huckaby JL, Perez JA, et al. Low-dose nitric oxide therapy for persistent pulmonary hypertension of the newborn. Clinical Inhaled Nitric Oxide Research Group. N Engl J Med 2000;342(7):469-474.

21. Roberts JD Jr, Fineman JR, Morin FC 3rd, Shaul PW, Rimar S, Schreiber MD, et al. Inhaled nitric oxide and persistent pulmonary hypertension of the newborn. The Inhaled Nitric Oxide Study Group. N Engl J Med 1997;336(9):605-610.

22. Kollef MH, Micek ST. Using protocols to improve patient outcomes in the intensive care unit: focus on mechanical ventilation and sepsis. Semin Respir Crit Care Med 2010;31(1):19-30.

This article is approved for Continuing Respiratory Care Education credit. For information and to obtain your CRCE

(free to AARC members) visit www.rcjournal.com

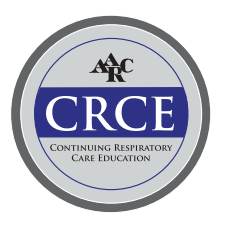

